\title{
Diseños óptimos bayesianos para estimación de parámetros en farmacocinética
}

\author{
Bayesian optimal designs for parameters estimation in
pharmacokinetics \\ Johnatan Cardona \\ jcardonj@unal.edu.co \\ Víctor Ignacio Lópezb \\ vilopez@unal.edu.co \\ Juan Carlos Correa $\mathrm{g}$
jccorrea@unal.edu.co
}

\section{Resumen}

En farmacología, particularmente en el campo farmacocinético, el interés fundamental es estudiar la concentración de un medicamento en plasma. En esta área usualmente se tienen modelos de tipo no lineal dadas las características particulares de administración del medicamento. Desde el enfoque bayesiano, el objetivo de construir diseños óptimos sujetos a una función de utilidad es maximizar la utilidad esperada asociada a algún funcional de interés para el investigador. En este trabajo se realiza una caracterización de los diseños óptimos obtenidos a través de dos funciones de utilidad asociadas a un criterio de optimalidad bayesiano (D-optimalidad bayesiano), para estimar en forma óptima los parámetros de dos modelos no lineales: 1) modelo monocompartimental con tasa de absorción y eliminación, 2) modelo bicompartimental con tasas de eliminación y absorción reversibles para el segundo compartimiento, ambos modelos bajo el supuesto de normalidad en los errores. Dicha caracterización se realizó vía simulación y con el fin de maximizar la función de utilidad se recurrió a la evolución diferencial.

Palabras clave: diseños bayesianos, evolución diferencial, farmacocinética, funciones de utilidad, modelos no lineales.

\footnotetext{
${ }^{a}$ Maestría en Ciencias - Estadística, Escuela de Estadística, Universidad Nacional de Colombia, Sede Medellín.

${ }^{\mathrm{b}}$ Profesor Asociado, Escuela de Estadística, Grupo de Investigación en Estadística, Universidad Nacional de Colombia, Sede Medellín.

${ }^{\mathrm{c}}$ Profesor Asociado. Escuela de Estadística, Grupo de Investigación en Estadística, Universidad Nacional de Colombia, Sede Medellín.
} 


\begin{abstract}
In pharmacology, especially on the pharmacokinetics field, the main interest is the study of the drug concentration in the plasma tissue. This area uses nonlinear models given by the particular administration of a medicine. The purpose of bayesian approach is to construct optimal designs restricted to an utility function, to maximize the expected utility associated to some functional in which the investigator is interested. In this work we made a characterization of the optimal designs obtained from two utility functions associated to an optimal bayesian criterion (Bayes D-optimality) to obtain optimal parameter estimates for two nonlinear models: 1) one-compartment model with absorption and elimination rate, 2) two-compartment model with absorption and elimination rates reversible for the second compartment, both models under normality assumption for the errors. The cited characterization was done via simulation and using differential evolution to maximize the utility.
\end{abstract}

Key words: Bayesian design, utility function, non-linear models, pharmacokinetics, diferential evolution.

\title{
1. Introducción
}

En algunas áreas de investigación interesa determinar, previo a la experimentación, cuáles son los niveles de la(s) variable(s) explicativa(s) que generan una estimación óptima de los parámetros asociados al modelo bajo estudio (López-Ríos \& RamosQuiroga 2007). Por ejemplo, en farmacocinética se requiere estudiar el proceso de acción de un fármaco en un organismo, ya sea humano, animal o vegetal, y para lograrlo se necesita conocer la frecuencia de administración, las dosis y los tiempos de muestreo $t_{1}, t_{2}, \ldots, t_{n}$, de tal manera que se pueda estimar en forma óptima algunos parámetros como el aclaramiento, la biodisponibilidad y la bioequivalencia, entre otros (Farmacología 2006). Es de anotar que los modelos usualmente involucrados en esta área son no lineales, lo cual dificulta la aplicación de la teoría clásica de diseños óptimos para modelos lineales, dado que la matriz de dispersión está expresada en función de los parámetros desconocidos que se desean estimar.

La teoría de diseños óptimos, en el contexto de la estadística bayesiana, presenta algunos resultados o criterios de optimalidad que pueden ser útiles para determinar las condiciones experimentales o los niveles de las covariables en los cuales se debe experimentar para obtener una estimación óptima de los parámetros de los modelos bajo estudio. Por ejemplo, Bernardo (1979) construye un criterio o función de utilidad a partir del concepto de información de Shannon, seleccionando el diseño que maximice la ganancia esperada en la información dada por un experimento. Spezzaferri (1989) halla los diseños óptimos usando un enfoque bayesiano con funciones de utilidad, y dichos diseños los emplea para la discriminación de modelos, la estimación de los parámetros y el problema dual de estimación y discriminación en el ámbito de los modelos lineales. Además, en Chaloner \& Verdinelli (1995) se 
realiza una revisión exhaustiva sobre la teoría de los diseños óptimos, presentando algunos criterios como A-optimalidad y D-optimalidad bayesiano tanto para modelos lineales como no lineales. En Merlé \& Mentré (1997) se realiza un análisis con tres algoritmos diferentes para optimizar un criterio de optimalidad bayesiano que estima en forma óptima los parámetros de un modelo farmacocinético (modelo de un compartimiento).

En este trabajo se caracterizan (vía simulación) los diseños óptimos obtenidos a partir de dos funciones de utilidad asociadas al criterio D-optimalidad bayesiano (diseño experimental bayesiano) para un modelo no lineal (modelo farmacocinético). Dichas funciones permiten determinar los tiempos de muestreo $t_{1}, t_{2}, \ldots, t_{n}$ que generan una estimación óptima, en términos de varianza, de los parámetros involucrados. El objetivo es caracterizar los diseños y determinar su comportamiento bajo diferentes escenarios de simulación (tamaños de muestra y niveles de correlación entre los parámetros).

\section{Diseño experimental bayesiano}

A continuación se presenta el diseño experimental bayesiano desde el marco de la teoría de la decisión. Primero se ilustra el criterio D-optimalidad bayesiano para el modelo lineal y posteriormente se presentan algunas aproximaciones asociadas al contexto de los modelos no lineales, los cuales son de interés en este trabajo. Lindley (1972) presenta una definición general del diseño experimental bayesiano desde el marco de la teoría de la decisión: un diseño $\xi$ debe ser seleccionado desde algún conjunto $\Xi$, y los datos $Y$ serán observados en un espacio muestral $\mathscr{Y}$. Basado en $Y$ alguna decisión $d$ será tomada desde un conjunto $\mathscr{D}$. La decisión consta de dos partes: primero la selección del diseño $\xi$ y a partir de este la elección de una decisión terminal $d, \theta$ representa el vector de parámetros desconocido asociado a un espacio parametral $\Theta$. Una función de utilidad general es de la forma: $U(d, \theta, \xi, Y)$. Para un diseño $\xi$, la utilidad esperada de la mejor decisión está dada por:

$$
U(\xi)=\int_{\mathscr{Y}} \operatorname{máx}_{d \in \mathscr{D}} \int_{\Theta} U(d, \theta, \xi, Y) P(\theta \mid Y, \xi) P(Y \mid \xi) d \theta d Y,
$$

donde $P(\cdot)$ denota una función de densidad de probabilidad con respecto a una medida apropiada. Según Chaloner \& Verdinelli (1995) el argumento de Lindley sugiere que una buena forma de diseñar experimentos es especificar una función de utilidad que refleje el propósito del experimento considerando la elección del diseño como un problema de decisión y seleccionar un diseño que maximice la utilidad esperada, además sugieren el enfoque de Lindley como una formulación unificada para la teoría del diseño experimental bayesiano. Las funciones de utilidad estudiadas en este trabajo parten de dicha idea, dada en la ecuación (11). 


\subsection{Criterio D-optimalidad bayesiano para el modelo lineal}

Sea $Y$ una variable aleatoria tal que $Y \mid \theta, \sigma^{2} \sim N\left(X \theta, \sigma^{2} I_{n}\right)$, donde la distribución a priori de $\theta \mid \sigma^{2}$ es $N\left(\theta_{0}, \sigma^{2} R^{-1}\right)$ con $\theta \in \mathbb{R}^{k}, R \in \mathbb{R}^{k \times k}, \sigma^{2}$ y $R$ son conocidas, se puede mostrar que la distribución posterior para $\theta$ es normal con media $\theta^{*}=$ $(n M(\xi)+R)^{-1}\left(X^{T} y+R \theta_{0}\right)$ y matriz de varianzas-covarianzas $\sigma^{2}(n M(\xi)+R)^{-1}$, donde $M(\xi)=\frac{1}{n} X^{T} X$ es la matriz de momentos.

Ahora se considera el problema de seleccionar un diseño $\xi$ para un modelo de regresión lineal normal

$$
E\left(Y \mid \theta, \sigma^{2}\right)=X \theta
$$

donde $\xi=\left[x_{1}, \ldots, x_{n}\right]$ representa las condiciones experimentales o los niveles de la(s) variable(s) independiente(s), las cuales permiten obtener una estimación óptima del vector de parámetros $\theta$.

Bernardo (1979), considera la ganancia esperada en la información de Shannon (1948) dada por un experimento como una función de utilidad, y se propone seleccionar un diseño que maximice la ganancia esperada de la información de Shannon, o equivalentemente, maximizar la distancia esperada de Kullback-Leibler (Gill 2000) entre la distribución a priori y la posterior de $\theta$ :

$$
\iint \log \left\{\frac{P(\theta \mid y, \xi)}{P(\theta)}\right\} P(y, \theta \mid \xi) d \theta d y .
$$

Como la distribución a priori no depende del diseño $\xi$, entonces el diseño que maximiza la ganancia esperada de Shannon es el que maximiza:

$$
U_{1}(\xi)=\iint \log \{P(\theta \mid y, \xi)\} P(y, \theta \mid \xi) d \theta d y
$$

Definiendo la función de utilidad (3) para el modelo (2) se obtiene:

$$
U_{1}(\xi)=-\frac{k}{2}-\frac{k}{2} \log (2 \pi)+\frac{1}{2} \log \operatorname{det}\left\{\sigma^{-2}(n M+R)\right\} .
$$

Maximizar la utilidad anterior es equivalente a maximizar la función:

$$
\phi_{1}(\xi)=\operatorname{det}\{n M(\xi)+R\},
$$

el cual es conocido como el criterio D-optimalidad bayesiano para el modelo lineal. Cuando se maximiza este criterio se maximiza la ganancia esperada de la información de Shannon o equivalentemente, se minimiza la varianza de la distribución posterior de $\theta$ (Chaloner \& Verdinelli 1995).

\subsection{Criterio D-optimalidad bayesiano para el modelo no-lineal}

En esta sección se considera el problema de seleccionar un diseño $\xi$ para un modelo no lineal. La búsqueda de los diseños óptimos se complica en este caso, dado que 
la ecuación (3) no tiene una solución analítica. Muchas de las aproximaciones que existen en la literatura para la utilidad esperada requieren de la aproximación normal para la distribución posterior. Varias aproximaciones a la normalidad son posibles (Berger 1985) e involucran la matriz de información de Fisher o la matriz de segundas derivadas del logaritmo de la verosimilitud o de la densidad posterior.

Sea $\hat{\theta}$ el estimador de máxima verosimilitud de $\theta$, una aproximación normal está dada por:

$$
\theta \mid Y, \xi \sim N\left(\hat{\theta},[n \psi(\hat{\theta}, \xi)]^{-1}\right)
$$

donde $[n \psi(\hat{\theta}, \xi)]$ es la matriz de información de Fisher esperada para un modelo que tiene: parámetros $\theta$ desconocidos, un diseño $\xi$ y un tamaño de muestra $n$. Se puede apreciar que la distribución posterior aproximada solo depende de los datos a través de $\hat{\theta}$.

Otra aproximación alternativa es:

$$
\theta \mid Y, \xi \sim N\left(\tilde{\theta},[R+n \psi(\hat{\theta}, \xi)]^{-1}\right)
$$

donde $\tilde{\theta}$ es la moda de la distribución posterior conjunta de $\theta$ (también llamado el estimador de máxima verosimilitud generalizado de $\theta$, (ver Berger (1985, página 133)) y $R$ es la matriz de segundas derivadas del logaritmo de la densidad a priori o la matriz de precisión de la distribución a priori (Chaloner \& Verdinelli 1995).

Aplicando la aproximación asociada a (17) en (3) se puede obtener la función de utilidad

$$
U_{1}(\xi)=-\frac{k}{2}-\frac{k}{2} \log (2 \pi)+\frac{1}{2} \int \log (\operatorname{det}\{n \psi(\theta, \xi)\}) p(\theta) d \theta
$$

De (9) se tiene que:

$$
\phi_{1}(\xi)=\int \log (\operatorname{det}\{n \psi(\theta, \xi)\}) p(\theta) d \theta
$$

como el criterio de optimalidad bayesiano.

Similarmente, el criterio de optimalidad obtenido usando (8):

$$
\phi_{1 R}(\xi)=\int \log (\operatorname{det}\{n \psi(\theta, \xi)+R\}) p(\theta) d \theta .
$$

Los criterios (10) y (11) se referencian como D-optimalidad bayesiano para el modelo no lineal (Chaloner \& Verdinelli 1995). Los diseños óptimos, en los contextos tanto lineal como no lineal, se caracterizan por ser modelos dependientes, es decir, antes de la experimentación se debe definir un modelo adecuado que se ajuste al experimento de interés. 


\section{Farmacocinética y el modelo de dos compartimientos}

La farmacocinética es la rama de la farmacología que estudia el paso de los medicamentos a través del organismo en función del tiempo y de la dosis. Comprende los procesos de absorción, distribución, metabolismo o biotransformación y excreción del medicamento. Algunos objetivos de la farmacocinética clínica son: desarrollar nuevos medicamentos, seleccionar la vía de administración, conocer la capacidad de acceso de los medicamentos a órganos y tejidos, establecer las vías metabólicas, caracterizar los procesos de eliminación, diseñar los regímenes de dosificación, entre otros (Farmacología 2006). Los modelos de compartimientos son de gran utilidad en farmacocinética, utilizados, entre otras aplicaciones, para modelar el nivel de concentración de un medicamento en la sangre de un individuo a lo largo del tiempo (Gabrielsson \& Weiner 2000). En la figura 1 y en la expresión (12) se ilustra el diagrama de dos compartimientos y el modelo asociado a la concentración en el compartimiento central, respectivamente.

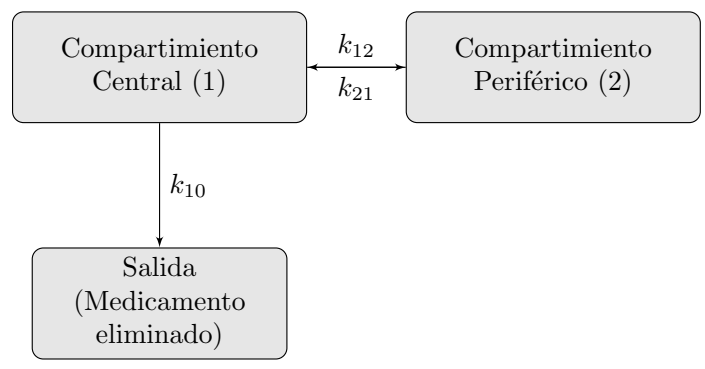

Figura 1: Diagrama asociado al modelo de dos compartimientos de un medicamento administrado por vía intravenosa

$$
Y_{1}(t)=A \cdot e^{-\alpha t}+B \cdot e^{-\beta t}+\varepsilon
$$

donde:

$$
\begin{gathered}
A=\frac{M_{0}}{V_{c}} \cdot \frac{\alpha-k_{21}}{\alpha-\beta} \\
B=\frac{M_{0}}{V_{c}} \cdot \frac{\beta-k_{21}}{\beta-\alpha} \\
\alpha=\frac{k_{21} \cdot k_{10}}{\beta} \\
\beta=\frac{1}{2}\left[k_{12}+k_{21}+k_{10}-\sqrt{\left(k_{12}+k_{21}+k_{10}\right)^{2}-4 k_{21} \cdot k_{10}}\right]
\end{gathered}
$$

Los parámetros asociados al modelo (12) $(A, B, \alpha$ y $\beta$ ) son llamadas macroconstantes, y pueden ser expresados en función de las constantes $k_{10}, k_{12}, k_{21}$ y 
$V_{c}$, representando, respectivamente, las tasas de eliminación y absorción reversibles para el segundo compartimiento y el volumen de distribución. Además $M_{0}$ denota la cantidad de dosis administrada, asumida conocida. El modelo anterior está considerado bajo el supuesto $\varepsilon \sim N\left(0, \sigma^{2}\right)$.

\section{Estudio de simulación}

Para caracterizar los diseños asociados al criterio D-optimalidad bayesiano para el modelo de dos compartimientos, dada en (12), se deben obtener diseños $\xi$ (tiempos de muestreo $\left.t_{1}, t_{2}, \ldots, t_{n}\right)$ que generen una estimación óptima de los parámetros, para lo cual se resuelven las expresiones (10) y (11) bajo diferentes tamaños de muestra $n$ (en este trabajo se consideran $n=2,3, \ldots, 7$ ) y diferentes niveles de correlación entre algunos parámetros del modelo anteriormente citado. Para calcular las integrales se recurrió a métodos de simulación y para maximizar la utilidad se utilizó el método de evolución diferencial (Storn \& Price 1997).

En cuanto a los escenarios de simulación para cada tamaño de muestra $(n=$ $2,3, \ldots, 7)$ se tuvieron en cuenta diferentes valores de $\rho: 0 \%, 10 \%, 30 \%, 50 \%$, $70 \%$ y $90 \%$, utilizados para definir los niveles de correlación entre $k_{10}, k_{12}$ y $k_{21}$. La distribución a priori seleccionada fue una normal multivariada con vector de medias $\mu=\left(\mu_{k_{10}}, \mu_{k_{12}}, \mu_{k_{21}}, \mu_{\tau}\right)$ y matriz de varianzas-covarianzas $\Sigma$. Para definir las estructuras de correlación en los diferentes escenarios, se empleó el enfoque adoptado por Jamshidian \& Schott (2007), quien define $\rho_{i j}=\rho^{|i-j|}$ :

$$
\rho=\left(\begin{array}{cccc}
\rho_{k_{10}} & \rho_{k_{10}, k_{12}} & \rho_{k_{10}, k_{21}} & \rho_{k_{10}, V_{d}} \\
\rho_{k_{12}, k_{10}} & \rho_{k_{12}} & \rho_{k_{12}, k_{21}} & \rho_{k_{12}, V_{d}} \\
\rho_{k_{21}, k_{10}} & \rho_{k_{21}, k_{12}} & \rho_{k_{21}} & \rho_{k_{21}, V_{d}} \\
\rho_{V_{d}, k_{10}} & \rho_{V_{d}, k_{12}} & \rho_{V_{d}, k_{21}} & \rho_{V_{d}}
\end{array}\right)=\left(\begin{array}{cccc}
1 & \rho^{1} & \rho^{2} & 0 \\
\rho^{1} & 1 & \rho^{1} & 0 \\
\rho^{2} & \rho^{1} & 1 & 0 \\
0 & 0 & 0 & 1
\end{array}\right),
$$

donde $\rho$ toma los valores definidos antes. En el estudio de simulación realizado por Jamshidian \& Schott (2007), se definen tres matrices de covarianza (con $\rho=0,0.5,0.8)$ con el fin de analizar el efecto de la correlación entre varias variables en una prueba de igualdad de matrices de covarianza en presencia de datos incompletos. En este trabajo se adoptó este enfoque con el fin de analizar el efecto de la correlación adaptándolo a los parámetros del modelo no lineal. También se pretende analizar el efecto de la matriz de covarianza sobre los diseños obtenidos por medio de los criterios (10) y (11). Para este modelo se tomó como información muestral los datos reportados en Davis et al. (2007) asociados a un experimento realizado con un tipo de medicamento llamado etodolac (la administración fue por 
vía intravenosa), a partir de los cuales se estimaron los hiper-parámetros:

$$
\begin{aligned}
\hat{\mu} & =\left(1.1\left(h^{-1}\right), 0.11\left(h^{-1}\right), 0.28\left(h^{-1}\right), 0.29(L / K g)\right) \\
\hat{\sigma}_{k_{10}}^{2} & =0.0169 \\
\hat{\sigma}_{k_{12}}^{2} & =0.000961 \\
\hat{\sigma}_{k_{21}}^{2} & =0.0025 \\
\hat{\sigma}_{V_{d}}^{2} & =0.0034
\end{aligned}
$$

Debido a la forma de administración del medicamento, no se tiene en cuenta el tiempo de rezago $\tau$, dado que el medicamento llega directamente al torrente sanguíneo y no es necesario un tiempo de espera para tomar la primera muestra. Como los datos originales no son reportados, se obtiene la región de diseño a partir de información a priori asociada a los hiper-parámetros estimados; la región es tal que $t \in[0,12]$ horas (Davis et al. 2007).

\subsection{Resultados}

A continuación se presentan los resultados asociados a los diseños óptimos obtenidos vía simulación en los diferentes escenarios definidos anteriormente. En principio se presenta la utilidad esperada bajo los criterios (10) y (11), los diseños para los diferentes tamaños de muestra y niveles de correlación entre algunos parámetros de los modelos de interés, y por último la evaluación de los diseños obtenidos por medio de la distribución del error cuadrático medio (ECM).

\subsubsection{Resultados modelo de dos compartimientos}
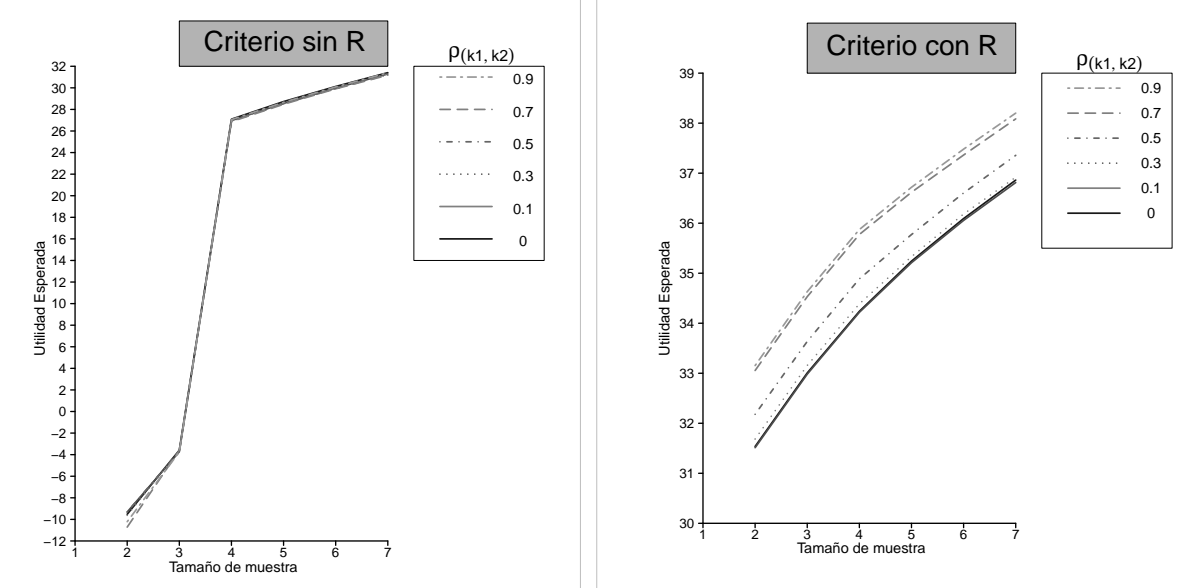

Figura 2: Utilidad esperada para los diferentes tamaños de muestra, bajo los criterios (10), (11) y con el modelo de dos compartimientos 
En la figura 2 se puede apreciar que el criterio (11) (criterio con la matriz de información a priori $R$ ) genera mayores utilidades en comparación al criterio (10) (criterio sin la matriz de información a priori $R$ ), para los diseños óptimos $\xi=$ $\left[t_{1}, t_{2}, \ldots t_{n}\right]$ asociados al modelo de dos compartimientos. Comparando la utilidad obtenida para iguales escenarios (tamaños de muestra y niveles de correlación), tanto para el modelo de un compartimiento como para el de dos compartimientos, el criterio (11) maximiza en mayor grado la información de Shannon o minimiza la varianza de la distribución posterior de los parámetros involucrados.

En las figuras 3 y 4 se puede apreciar la estructura de los diseños óptimos bajo los criterios (10) (criterio sin la matriz de información a priori $R$ ) y (11) (criterio con la matriz de información a priori $R$ ) asociados al modelo de dos compartimientos. Los diseños bajo el criterio (10), presentan un comportamiento similar bajo los diferentes niveles de correlación en cada uno de los tamaños de muestra. $\mathrm{Al}$ parecer, el nivel de correlación entre las constantes de absorción y eliminación $\left(k_{10}, k_{12}\right.$ y $\left.k_{21}\right)$ del modelo de dos compartimientos (modelo (12) no afectan la estructura general de los diseños bajo el criterio (10). Para los diseños obtenidos bajo el criterio (11) se puede apreciar que para tamaños de muestra entre dos y cuatro tiempos, la estructura de los diseños es similar bajo los diferentes niveles de correlación. Algo diferente sucede al considerar tamaños de muestra entre cinco y siete tiempos, ya que cuando se consideran niveles de correlación mayores al $50 \%$ los diseños tienden a concentrarse al comienzo de la región de diseño entre 0 y 0.79 horas. Para el criterio (11), bajo el modelo de dos compartimientos, la correlación tiene efecto sobre la estructura de los diseños cuando se consideran tamaños de muestra mayores o iguales a cinco tiempos.

En la figura 3 se puede apreciar de nuevo el comportamiento particular del criterio (11), el cual tiende a encontrar una mayor cantidad de tiempos de muestreo al comienzo de la región de diseño.

En las figuras 5 y 6 se puede apreciar en detalle el comportamiento o la forma de los criterios para los diseños de dos puntos. Para el modelo de dos compartimientos también se puede ver el mismo comportamiento del criterio D-optimalidad bayesiano en relación a la forma de la superficie, la cual es totalmente caótica en el caso donde no se considera la matriz de precisión $R$ (gráficos en la columna izquierda de las figuras (5 y 60) y totalmente suave cuando se considera la matriz de precisión (criterio 11). También se puede apreciar que para los tres diferentes niveles de correlación la forma del criterio es básicamente la misma, lo cual es coherente con lo observado en la estructura de los diseños óptimos de dos tiempos apreciados en la figura 3 , 


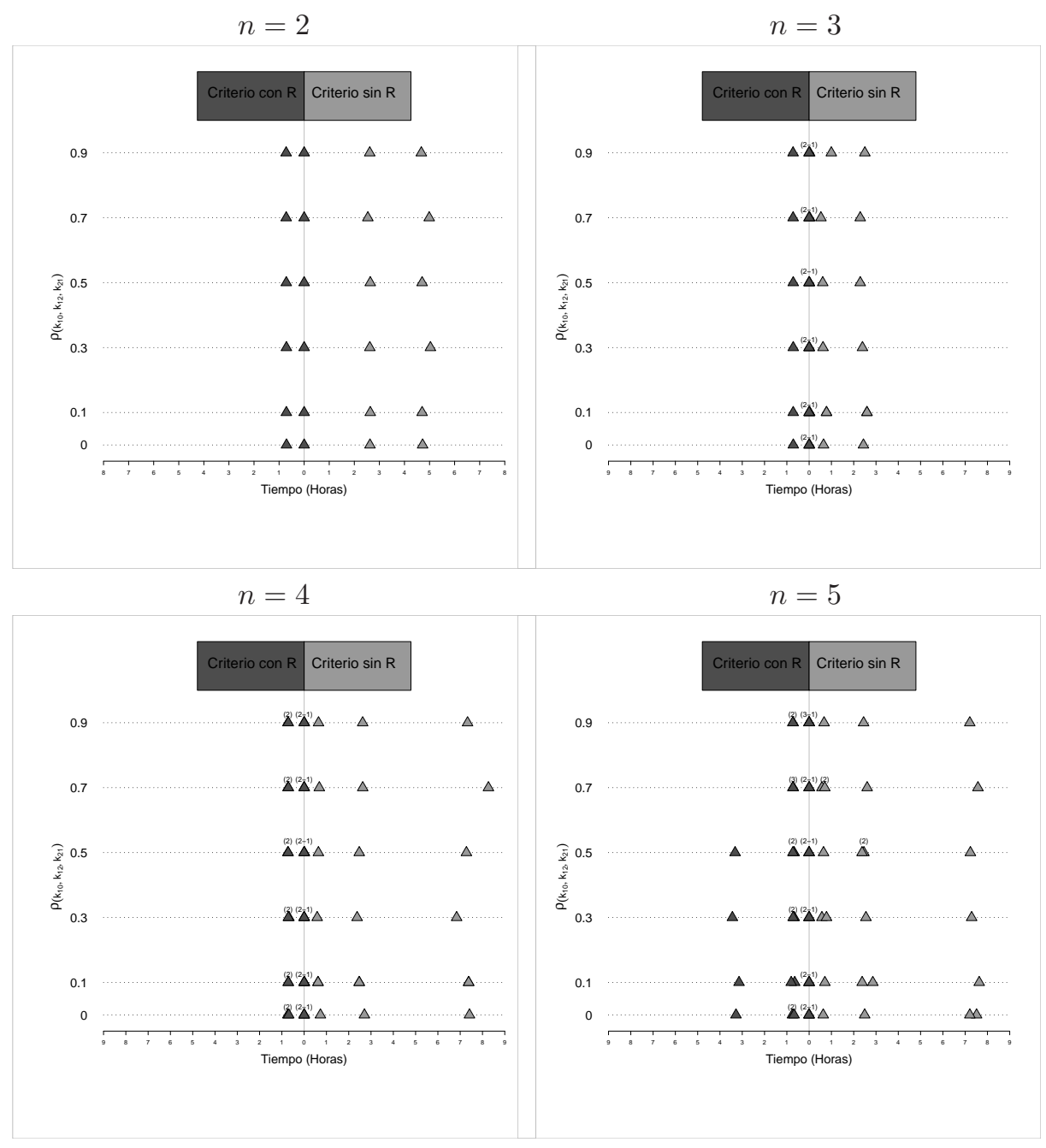

Figura 3: Comparación de diseños óptimos con diferentes tamaños de muestra $(n=$ $1, \ldots, 7)$ y estructuras de correlación $\rho_{\left(k_{10}, k_{12}, k_{13}\right)}$, obtenidos con los criterios (10), (11) en el modelo de un compartimiento. Las etiquetas $(\cdot)$ y $(\cdot-\cdot)$ hacen referencia al número de observaciones en un mismo tiempo. 


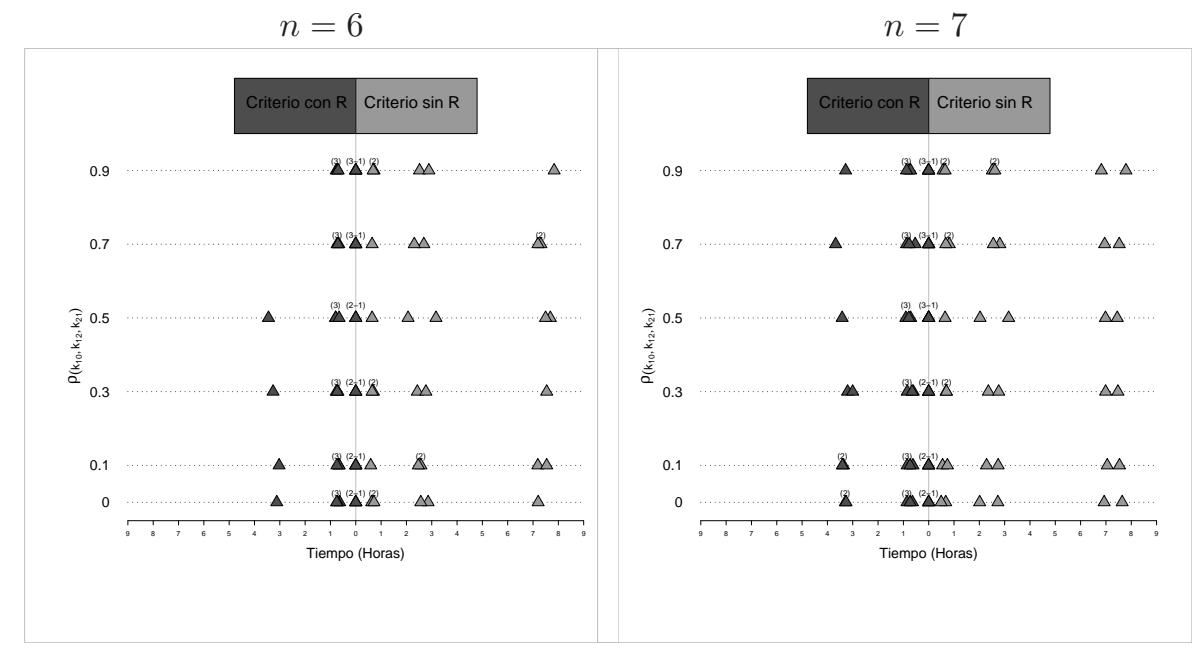

Figura 4: Comparación de diseños óptimos con diferentes tamaños de muestra $(n=$ $1, \ldots, 7)$ y estructuras de correlación $\rho_{\left(k_{10}, k_{12}, k_{13}\right)}$, obtenidos con los criterios (10), (11) en el modelo de un compartimiento. Las etiquetas $(\cdot)$ y $(\cdot-\cdot)$ hacen referencia al número de observaciones en un mismo tiempo.

\section{Conclusiones}

A partir del estudio de simulación realizado en este trabajo, con el fin de caracterizar los diseños óptimos obtenidos a partir del criterio D-optimalidad bayesiano para el modelo de dos compartimientos, se concluye lo siguiente:

- Para el modelo de dos compartimientos la utilidad obtenida bajo los diferentes escenarios (tamaños de muestra y niveles de correlación) con el criterio (11) (criterio con información a priori $R$ ) fue mucho mayor en comparación a la obtenida bajo el criterio (10) (ver figura2). Otra apreciación interesante, es la diferencia en el comportamiento de la utilidad esperada entre los criterios (10) y (11) (ver figuras 5 y [6), ya que cuando no se considera la matriz de precisión $R$ en el criterio de optimalidad, la superficie es totalmente caótica, pero cuando sí se considera dicha matriz, la superficie es totalmente suave; existe una gran diferencia cuando se tiene en cuenta la información a priori (a través de $R$ ) en el criterio D-optimalidad bayesiano.

- La estructura de los diseños óptimos para el modelo de dos compartimientos, bajo el criterio sin información a priori (criterio sin $R$ ) presenta un comportamiento similar bajo los diferentes niveles de correlación entre las tasas $k_{10}$, $k_{12}$ y $k_{21}$. Para este modelo, bajo el criterio sin $R$, considerar o no la correlación entre dichos parámetros no tiene impacto sobre la estructura de los diseños óptimos. Algo diferente ocurre con la estructura de los diseños óptimos para el modelo de dos compartimientos bajo el criterio con información a priori (criterio con $R$ ), porque para tamaños de muestra entre dos y 

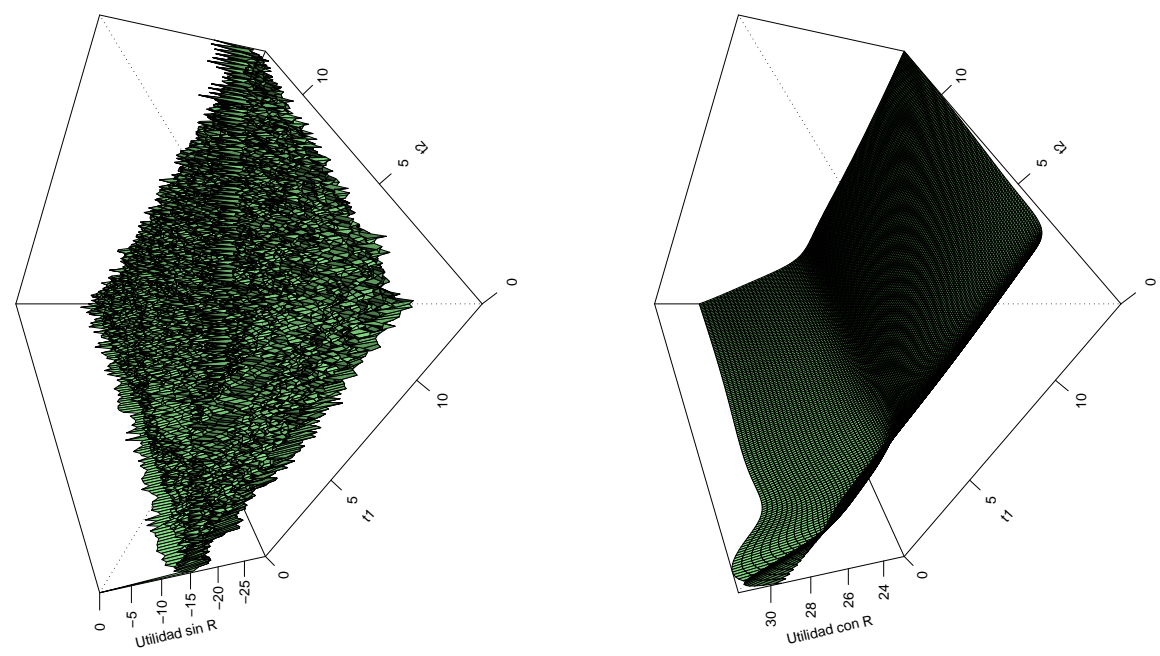

Figura 5: Superficies de los criterios (10) y (11) asociadas al modelo de dos compartimientos para el nivel de correlación de $0 \%$ entre $k_{10}, k_{12}$ y $k_{21}$. 

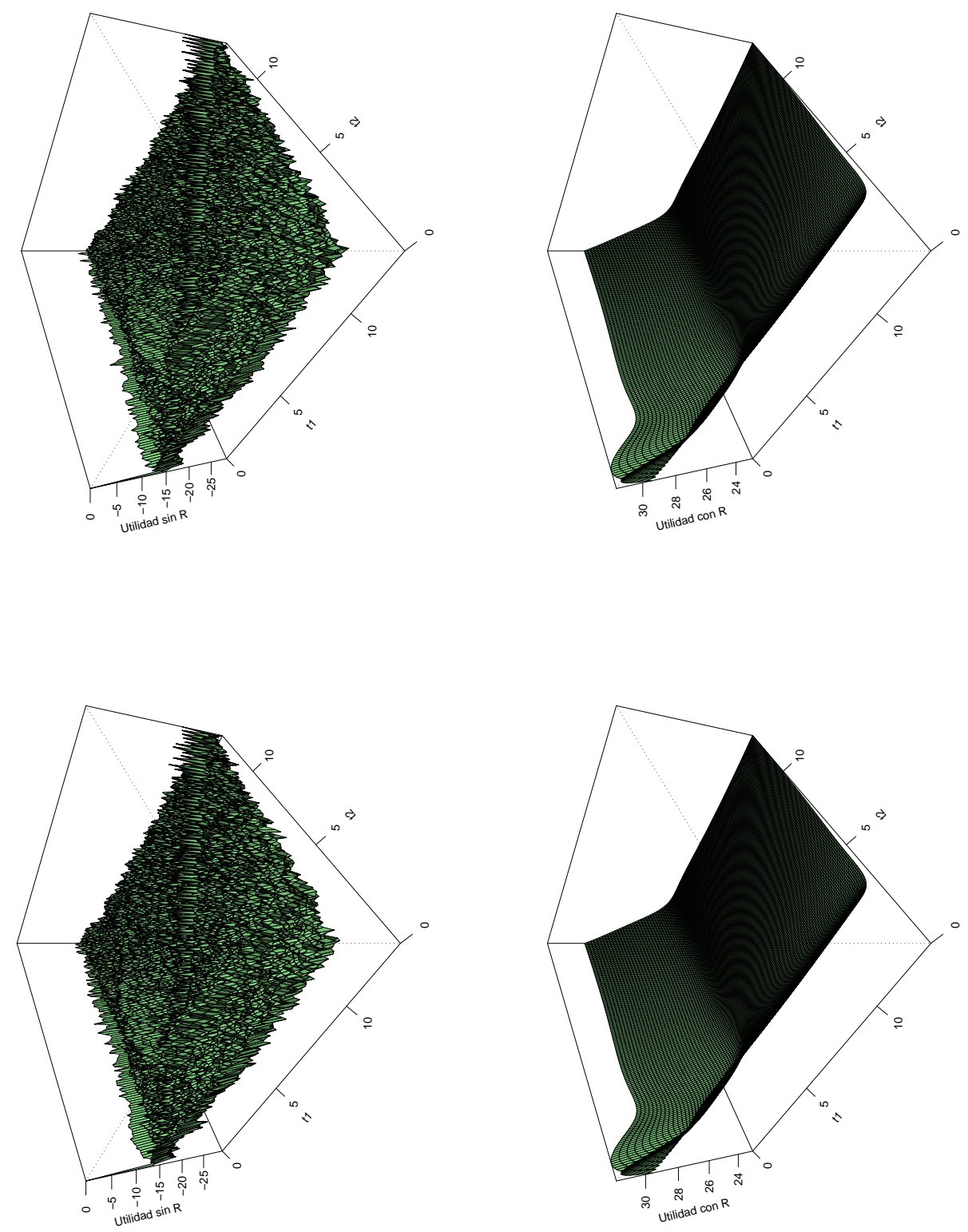

Figura 6: Superficies de los criterios 10 y 11 en el modelo de dos compartimientos para los niveles de correlación al $30 \%$ y $50 \%$ entre $k_{10}, k_{12}$ y $k_{21}$.

Comunicaciones en Estadística, junio 2012, Vol. 5, No. 1 
cuatro tiempos la estructura de los diseños es similar bajo todos los niveles de correlación, pero cuando se consideran tamaños de muestra entre cinco y siete tiempos los niveles de correlación altos $(>70 \%)$ entre las tasas $k_{10}$, $k_{12}$ y $k_{21}$ sí generan influencia en la estructura de los diseños.

- Un aspecto sobresaliente en este trabajo, fue la combinación de la metodología de evolución diferencial con la programación en paralelo, para optimizar ambos criterios bayesianos (criterio D-optimalidad con $R$ y $\sin R$ ) bajo los modelos de uno y dos compartimientos. Se aprovecharon las ventajas de la evolución diferencial para optimizar funciones complejas como las asociadas al criterio D-optimalidad bayesiano y se redujeron significativamente los tiempos de cálculo asociados a la múltiple evaluación de la función objetivo al paralelizar las rutinas implementadas en $\mathbf{R}$.

\section{Agradecimientos}

A los jurados por sus comentarios y sugerencias. A la Escuela de Estadística y a la Facultad de Ciencias de la Universidad Nacional de Colombia, Sede Medellín por su apoyo para la realización de este trabajo.

Recibido: 8 de marzo de 2011 Aceptado: 1 de septiembre de 2011

\section{Referencias}

Berger, J. (1985), Statistical Decision Theory and Bayesian Analysis, Springer, New York.

Bernardo, J. (1979), 'Expected information as expected utility', The Annals of Statistics 7(3), 686-690.

Beyer, H. G. \& Schwefel, H. P. (2002), 'Evolution strategies a comprehensive introduction', Natural Computing pp. 3-52.

Chaloner, K. \& Verdinelli, I. (1995), 'Bayesian experimental design: A review', Statistical Science 10, 379-423.

Davis, J. L., Papich, M. G., Morton, J. \& Gayle, A. T. (2007), 'Pharmacokinetics of etodolac in the horse following oral and intravenous administration', vet. Pharmacol 30, 43-48.

Farmacología (2006), Argentina.

*http://med.unne.edu.ar/catedras/farmacologia/temasfarma/volumen1/cap2farmacocinet.pdf 
Gabrielsson, J. \& Weiner, D. (2000), Pharmacokinetic and Pharmacodynamic Data Analysis, Swedish Pharmaceutical Press.

Gill, J. (2000), Bayesian Methods, Chapman and Hall.

Jamshidian, M. \& Schott, J. R. (2007), 'Testing equality of covariance matrices when data are incomplete', Computational Statistics and Data Analysis pp. 4227-4239.

Lindley, D. (1972), Bayesian Statistic - A review, SIAM.

López-Ríos, V. I. \& Ramos-Quiroga, R. (2007), 'Introducción a los diseños óptimos', Revista Colombiana de Estadística 30(1), 37-51.

Merlé, Y. \& Mentré, F. (1997), 'Stochastic optimization algorithms of a bayesian design criterion for bayesian parameter estimation of nonlinear regression models: Application in pharmacokinetics', Mathematical Biosciences 144, 4577.

R (2010), R-project, Vienna, Austria. *http://www.R-project.org

Shannon, C. (1948), 'A mathematical theory of communication', Bell System Technical Journal 27(3), 379-423.

Spezzaferri, F. (1989), 'Nonsequential designs for model discrimination and parameter estimation', Bayesian Statistics 3, 777-783.

Storn, R. \& Price, K. (1997), 'Differential evolution: A simple and efficient heuristic for global optimization over continuous spaces', Journal of Global Optimization 11, 341-359.

\section{A. Anexos}

\section{Evolución diferencial}

La evolución diferencial fue propuesta por estudiantes de Technical University of Berlin (TUB) (Beyer \& Schwefel 2002). Estos son métodos de búsqueda basados en la idea de la evolución natural de los seres vivos y presentan ventajas con respecto a los métodos tradicionales de optimización. En especial, estos métodos iterativos convergen al óptimo global de la función objetivo de interés, sin importar su grado de complejidad y su dominio, evitando procedimientos adicionales que en ocasiones se desvían hacia óptimos locales. Desde un enfoque genético, se parte de un conjunto de individuos progenitores, los cuales son mutados y posteriormente cruzados, generándose nuevos individuos con aquellas características que más dominaron en los progenitores. 


\section{Programación en paralelo con $\mathbf{R}$}

La programación en paralelo es una manera de realizar varios cálculos de manera simultánea. En la forma tradicional de programar los cálculos se realizan en serie, lo cual en ocasiones puede ser desfavorable por los altos tiempos de espera. Cuando se programa una tarea en paralelo, dicha tarea se distribuye a varios procesadores (físicos o virtuales), los cuales por separado realizan la parte que les corresponde. En cambio, cuando se programa en serie, toda la tarea es asignada a un procesador. Existen tres escenarios para realizar un cálculo en paralelo:

- Varios procesadores en un mismo chip (procesadores virtuales).

- Varios chips en un mismo computador (procesadores físicos).

- Varios computadores conectados en una red o cluster.

En $R$ existen varias formas de realizar la paralelización. Entre las más efectivas se encuentran:

- snow: se basa en códigos vectorizados por medio de funciones como apply () para realizar la división de las tareas.

- foreach: usa un bucle for para realizar la división en las tareas.

- multicore: es adecuado para hardware de varios núcleos.

Uno de los problemas abordados en este trabajo fue la maximización de algunas funciones bastante complejas (ecuaciones (10), (11)), lo cual se logró por medio de algoritmos evolutivos. Una de las desventajas de este método es la gran cantidad de evaluaciones que se tienen que hacer sobre la función objetivo. Para superar este problema se recurrió a la programación en paralelo anteriormente citada. 